\title{
Neuroanatomy of Fragile X Syndrome Is Associated with Aberrant Behavior and the Fragile X Mental Retardation Protein (FMRP)
}

\author{
Doron Gothelf, MD ${ }^{1,2}$, Joyce A. Furfaro, $\mathrm{PhD}^{3}$, Fumiko Hoeft, MD, PhD ${ }^{3}$, Mark A. Eckert, \\ $\mathrm{PhD}^{4}$, Scott S. Hall, $\mathrm{PhD}^{3}$, Ruth O'Hara, $\mathrm{PhD}^{5}$, Heather W. Erba, $\mathrm{PhD}^{3}$, Jessica Ringel, $\mathrm{BS}^{3}$, \\ Kiralee M. Hayashi, BS ${ }^{6}$, Swetapadma Patnaik, MS $^{3}$, Brenda Golianu, MD ${ }^{7}$, Helena C. \\ Kraemer, PhD $^{5}$, Paul M. Thompson, PhD ${ }^{6}$, Joseph Piven, $\mathbf{M D}^{8}$, and Allan L. Reiss, $\mathbf{M D}^{3}$ \\ ${ }^{1}$ Behavioral Neurogenetics Center, Child Psychiatry Department, Schneider Children's Medical \\ Center of Israel, Petah Tiqwa \\ ${ }^{2}$ Sackler Faculty of Medicine, Tel Aviv University, Tel Aviv, Israel \\ ${ }^{3}$ Center for Interdisciplinary Brain Sciences Research, Department of Psychiatry and Behavioral \\ Sciences, Stanford University School of Medicine, Stanford, CA \\ ${ }^{4}$ Department of Otolaryngology-Head and Neck Surgery, Medical University of South Carolina, \\ Charleston, SC \\ ${ }^{5}$ Department of Psychiatry and Behavioral Sciences, Stanford University School of Medicine, \\ Stanford, CA \\ ${ }^{6}$ Laboratory of Neuroimaging, Department of Neurology, University of California Los Angeles School \\ of Medicine, Los Angeles \\ ${ }^{7}$ Department of Anesthesia, Stanford University School of Medicine, Stanford, CA \\ ${ }^{8}$ Department of Psychiatry, University of North Carolina, Chapel Hill, NC
}

\section{Abstract}

Objective-To determine how neuroanatomic variation in children and adolescents with fragile $\mathrm{X}$ syndrome is linked to reduced levels of the fragile $\mathrm{X}$ mental retardation-1 protein and to aberrant cognition and behavior.

Methods-This study included 84 children and adolescents with the fragile $\mathrm{X}$ full mutation and 72 typically developing control subjects matched for age and sex. Brain morphology was assessed with volumetric, voxel-based, and surface-based modeling approaches. Intelligence quotient was evaluated with standard cognitive testing, whereas abnormal behaviors were measured with the Autism Behavior Checklist and the Aberrant Behavior Checklist.

Results-Significantly increased size of the caudate nucleus and decreased size of the posterior cerebellar vermis, amygdala, and superior temporal gyrus were present in the fragile $\mathrm{X}$ group. Subjects with fragile $\mathrm{X}$ also demonstrated an abnormal profile of cortical lobe volumes. A receiver operating characteristic analysis identified the combination of a large caudate with small posterior cerebellar vermis, amygdala, and superior temporal gyrus as distinguishing children with fragile $\mathrm{X}$ from control subjects with a high level of sensitivity and specificity. Large caudate and small posterior

\footnotetext{
(C) 2007 American Neurological Association

Address correspondence to Prof Reiss, Center for Interdisciplinary Brain Science, Department of Psychiatry and Behavioral Sciences, Stanford University Medical Center, 401 Quarry Road, Stanford, CA 94305-5795. reiss@ stanford.edu.

This article includes supplementary materials available via the Internet at

http://www.interscience.wiley.com/jpages/0364-5134/supp-mat
} 
cerebellar vermis were associated with lower fragile $\mathrm{X}$ mental retardation protein levels and more pronounced cognitive deficits and aberrant behaviors.

Interpretation-Abnormal development of specific brain regions characterizes a neuroanatomic phenotype associated with fragile X syndrome and may mediate the effects of FMRl gene mutations on the cognitive and behavioral features of the disorder. Fragile $\mathrm{X}$ syndrome provides a model for elucidating critical linkages among gene, brain, and cognition in children with serious neurodevelopmental disorders.

Cognitive dysfunction and aberrant behaviors are the hallmarks of childhood neurodevelopmental disorders. Though current diagnostic constructs for these disorders are useful for professional communication and obtaining therapeutic services, the current taxonomic categories are likely to be too heterogeneous to support scientific investigation. ${ }^{1}$ More homogeneous models are needed to overcome the problem of heterogeneity in our current taxonomy of neurodevelopmental disorders, so that genetic and neural mechanisms underlying the development and course of maladaptive cognition and behavior can be determined. ${ }^{2}$

Fragile $\mathrm{X}$ syndrome ( $\mathrm{FraX}$ ) is caused by a mutation in the FMR1 gene on chromosome $\mathrm{Xq} 27.3$; it is the most common known cause of inherited neurodevelopmental disability. The FMRI gene codes for the fragile X mental retardation protein (FMRP) that regulates the translation of numerous proteins critical for brain maturation and function. ${ }^{3}$ Lack of FMRP leads to dendritic spine dysmorphogenesis and impaired synaptic plasticity. ${ }^{3}$ Both male and female individuals with the FMRI full mutation show intellectual deficits, with typical cognitive level in the moderate-to-severe range of mental retardation in male individuals, and low average intelligence quotient (IQ) in female individuals. ${ }^{4}$ The most prominent neurobehavioral phenotype associated with FraX is autistic-spectrum behaviors seen in 60 of $90 \%$ of male individuals and 25 to $80 \%$ of female individuals. ${ }^{5}$ Autistic behaviors include impairments in social interaction and communication, and repetitive/stereotypic behaviors. ${ }^{4}$

Previous structural brain imaging studies indicate that abnormalities of brain morphology occur in FraX (see Hessl and colleagues ${ }^{6}$ for review). In brief, in the context of overall normal brain size, individuals with FraX have excessive caudate nucleus $(\mathrm{CN})$ size and small superior temporal gyrus (STG) and posterior (cerebellar) vermis (PV). Abnormalities in size of both the $\mathrm{CN}$ and PV correlate with IQ and FMRP levels. ${ }^{7,8}$ Furthermore, results from diffusion tensor and functional imaging studies indicate that there is abnormal anterior cerebral-caudate connectivity and function in FraX. ${ }^{9-11}$ FMRP levels also correlate with anterior cerebral and $\mathrm{CN}$ activation during specific functional magnetic resonance imaging tasks in individuals with FraX. ${ }^{11}$

As a causatively homogeneous disorder where affected children share a common genetic risk factor, FraX is a valuable model from which to learn about pathways leading from specific gene mutation to aberrant brain development and cognitive-behavioral symptoms. ${ }^{2}$ Accordingly, the aim of this study was to determine the associations among levels of FMRP, neuroanatomic variation, and aberrant cognition and behavior in FraX using multiple complementary image processing and statistical approaches. Based on previous imaging and behavioral findings in $\mathrm{FraX}^{6-8}$ and autism, ${ }^{12}$ we hypothesized that abnormal morphology of the CN, PV, amygdala, and STG would most strongly distinguish between FraX and control subjects. We further predicted that abnormal morphology would be associated with FMRP levels, IQ, and aberrant behaviors. Though previous studies have identified some neuroanatomic abnormalities in FraX and their association with FMRP levels and IQ, ${ }^{7,8,13}$, 14 this study is novel in several aspects: (1) it uses three-dimensional high-resolution image datasets; (2) new image processing techniques are employed (ie, voxel brain morphometry [VBM] and surface-based modeling); (3) we utilize a novel statistical approach to image 
analysis (the Quality Receiver Operating Characteristic Curve [QROC]); and (4) it is the largest sample of FraX subjects published to date.

\section{Subjects and Methods}

\section{Subjects}

The study sample consisted of 84 children with FraX and 72 age- and sex-matched healthy control subjects. Children with FraX were recruited through advertisements in the National FraX Foundation and through Medical Genetic Divisions, whereas healthy control subjects were recruited through newsletters to schools and to athletic leagues. Control subjects underwent psychiatric screening using the parent version of the Child Behavior Checklist. ${ }^{15}$ Exclusion criteria for control subjects included: (1) a score of more than 1 standard deviation from the mean on any of the Child Behavior Checklist factors, (2) past or present psychiatric disorder (excluding preschool simple phobias), (3) past or present neurological disorder, (4) past or present special education intervention, (5) history of alcohol or substance use, and (6) history of any sensory deficit such as hearing or vision loss. Exclusion criteria for subjects with FraX included: (1) current seizure disorder or the presence of other neurological symptoms not commonly associated with fragile X, (2) hearing or vision deficits, and (3) presence of psychiatric disorders not commonly associated with fragile $\mathrm{X}$ (eg, psychotic disorder).

Demographic information about the groups and medical and behavioral characteristics of the FraX group are presented in Tables 1 and 2. As expected in an X-linked disorder, there was a significant difference in full-scale IQ (FSIQ) between male subjects with FraX, female subjects with FraX, and control subjects. Only 18 subjects with FraX, 21.4\% of the sample, had participated in a previous brain imaging study. $8,13,16$

The study protocols were approved by the human subjects committees at Stanford University School of Medicine and Johns Hopkins University School of Medicine. Assent was obtained from all subjects, and written consent was obtained from their parents or guardians.

\section{DNA and Protein Analyses}

Blood samples were collected from all participants with FraX to confirm the genetic diagnosis. Blood analyses were performed by Kimball Genetics (Denver, CO). The diagnosis of FraX was confirmed by DNA analysis. Standardized Southern blot and polymerase chain reaction analyses were performed followed by $F M R-1$ - specific probe hybridization. ${ }^{17}$ The CGG repeat number was calculated from the Southern blot autoradiogram images. FMRP levels were ascertained by calculating the percentage of peripheral lymphocytes containing FMRP using immunostaining techniques. ${ }^{18}$ All female subjects with FraX carried the full mutation. Of the 45 male subjects with FraX, 6 (12.8\%) displayed a mosaic pattern; that is, they carried both methylated (full mutation) and unmethylated (premutation) amplifications at the FraX site.

\section{Cognitive-Behavioral Assessment}

All subjects underwent age-appropriate cognitive testing to obtain an FSIQ score (see supplementary material for details). The presence of autistic symptoms in subjects with FraX was measured with the Autism Behavior Checklist $(\mathrm{ABC}),{ }^{19}$ whereas maladaptive behaviors associated with developmental disability were measured with the Aberrant Behavior Checklist $(\mathrm{AbBC}) .{ }^{20}$ Higher scores on the $\mathrm{ABC}$ and $\mathrm{AbBC}$ indicate greater impairment.

\section{Image Acquisition and Processing}

General Electric 1.5-Tesla Signa Scanners (GE Imaging Systems, Milwaukee, WI) were used to obtain magnetic resonance images. Scans were obtained from 1992 through 2003 at either Stanford or Johns Hopkins medical centers. The number of subjects scanned in each group was 
comparable across imaging sites (Stanford: 51 FraX and 39 control subjects; Hopkins: 33 FraX and 33 control subjects; $\chi^{2}=0.41 ; p=0.41$ ). However, more male relative to female subjects were scanned at Stanford (44 male and 46 female subjects) than at Hopkins (44 male and 22 female subjects) $\left(\chi^{2}=4.9 ; p=0.027\right)$. The average age of subjects scanned at Stanford (13.4 \pm 4.9 years) was also older than those imaged at Hopkins $(9.4 \pm 4.6$ years; $p<0.0001)$.

Identical coronal three-dimensional volumetric spoiled gradient echo series were obtained at both sites with parameters that remained consistent throughout the study: $\mathrm{TR}=35$ milliseconds, $\mathrm{TE}=6$ milliseconds, flip angle $=45^{\circ}$, slice thickness $=1.5$, resolution $(x, y, z)=0.94 \times 0.94$ $\times 1.50$, and acquisition matrix size $=256 \times 192$ for 124 contiguous slices.

Details regarding data processing steps are described in the supplementary material. In brief, volumetric analysis was conducted following well-established protocols that included removal of nonbrain tissues from the images, correction of equipment-related image artifacts, and segmentation of tissue components (gray, white, cerebrospinal fluid) and anatomically distinct regions (cerebral lobes, anterior cerebral cortex, subcortical regions). We chose to focus on gray matter (GM) in our volumetric analyses because FMRP is a neuronal protein primarily localized in dendrites and cell bodies. These protocols have been shown to be robust and reliable in a previous study of anatomic data collected from multiple scan sites using the same pulse sequence. ${ }^{21}$ Raters were blind to subject diagnosis, sex, and other subject characteristics. For VBM, optimized $\mathrm{VBM}^{22}$ and tensor-based morphometry (TBM) ${ }^{23}$ analyses were performed using Statistical Parametric Mapping 2 (SPM2) software (http://www.fil.ion.ucl.ac.uk/spm). Surface-based modeling of the $\mathrm{CN}^{24}$ was used as a method to confirm and further specify volumetric and VBM results. Assessment of intersite scan compatibility is provided in the supplementary material.

\section{Statistical Analysis}

VOLUMETRIC—Data were first examined for normality to conform to the assumption of the parametric statistics employed. To reduce the possibility of type 1 error, we used multivariate analyses of variance (MANCOVAs) using anatomically related groups of dependent variables (eg, subcortical regions) as the initial method for between-group comparisons. Group and sex were used as fixed effects, and age and total brain tissue as covariates in all analyses. Imaging site (Stanford/Hopkins) was also included in each analysis to remove potential effects associated with this variable. When significant effects were observed for the MANCOVA (Bonferroni corrected Wilks' lambda, $p<0.01$ ), univariate analyses of covariance (ANCOVAs) were conducted to evaluate group differences in single region volumes. The interaction terms Group $\times$ Sex or Group $\times$ Imaging Site were retained in the models when the analyses provided a $p$ value less than 0.1 . Asymmetry measures were performed with a repeated-measures analysis of variance; $p<0.05$ for the Group $\times$ Side (right/left) interaction was considered evidence of between-group differences in asymmetry.

VOXEL BRAIN MORPHOMETRY-TBM was performed to identify local shape differences between FraX and control groups that might affect results from VBM analysis. This analysis was performed because voxel modulation to examine subcortical GM volume could introduce effects of shape deformation caused by larger adjacent lateral ventricles in the FraX population. ${ }^{8}$ TBM analysis confirmed ventricular shape differences between the groups using a family-wise error correction at a significance level of 0.05 (peak: $x=0, y=8$, and $z=$ 14). FraX was compared with control subjects using ANCOVA, with the principal eigenvariate from the TBM analysis, brain tissue volume, sex, age, and scan site (Stanford, Hopkins) as covariates of uninterest ( $p=0.05$ family-wise error corrected, extent threshold 10). 
SURFACE-BASED MODELING-Statistical maps were generated indicating local group differences in radial $\mathrm{CN}$ volume. The local $p$ value was plotted onto the surface at each point showing between-group differences; overall $p$ values were assigned to the maps using permutation testing to give an overall significance value for the effect, adjusting for multiple comparisons. ${ }^{24}$

\section{QUALITY RECEIVER OPERATING CHARACTERISTIC CURVE ${ }^{25}$-The QROC} (v4.19; http://mirecc.stanford.edu) was applied to identify the brain regions that most sensitively and specifically distinguished subjects with FraX from control subjects. Only regions that were shown to be significantly different between groups in the volumetric analyses described earlier were entered into the QROC (see supplementary material).

ASSOCIATIONS BETWEEN VARIABLES-Scores on the ABC and the AbBC were standardized by $Z$-score transformation of the FraX sample data. To investigate the role of the $\mathrm{CN}, \mathrm{PV}$, amygdala, and STG in mediating the cognitive and behavioral phenotype in FraX, we conducted a series of exploratory correlation analyses between the size of these regions (statistically adjusted for brain tissue volume) and FMRP levels, FSIQ, ABC, and AbBC scores. The nonparametric Spearman's $\rho$ correlation was used as male subjects with FraX tended to cluster at the lower end of the IQ and FMRP measures in these analyses. All $p$ values reported are two-sided.

\section{Results \\ Volumetric Analysis}

There were no significant differences between groups or a significant group by sex interaction for total brain volume. MANCOVAs were performed separately for the following five groups of related brain regions: (1) cortical lobes GM, (2) subcortical regions (CN, lenticular nucleus, and thalamus), (3) temporal lobe regions (STG GM, amygdala, and hippocampus), (4) anterior cerebral regions GM (dorsal, midsuperior, midinferior, and ventral), and (5) cerebellum (total cerebellar tissue, anterior vermis, PV). Except as noted later, both age and total brain tissue volume were significantly correlated with all dependent measures of interest. The interaction between group and imaging site was not significant for any of the five MANCOVAs (Table $3)$.

CORTICAL LOBES-The MANCOVA was significant for group. Follow-up ANCOVAs indicated that the FraX group had significantly smaller frontal and temporal and significantly larger parietal and occipital GM volumes relative to control subjects.

SUBCORTICAL REGIONS-The MANCOVA was significant for group. The interaction term, Group $\times$ Sex, was included in the final model $(p=0.002)$. Follow-up ANCOVAs showed $\mathrm{CN}$ volume to be significantly larger in the FraX group (Fig. 1). The increase in $\mathrm{CN}$ volume was $23.4 \%$ for male and $8.8 \%$ for female subjects. Thalamus volume was also significantly enlarged for the FraX group, though less so than the caudate. Lenticular nucleus volumes were not significantly different between the groups.

TEMPORAL LOBE REGIONS-The MANCOVA was significant for group. Follow-up ANCOVAs indicated that the FraX group had significantly smaller STG GM and amygdala volumes.

ANTERIOR CEREBRAL REGIONS-The MANCOVA was significant for group. Followup ANCOVAs indicated that the FraX group had significantly reduced anterior ventral and 
anterior midinferior cerebral GM, and significantly increased anterior dorsal cerebral GM volumes.

CEREBELLUM-The MANCOVA was significant for group. Age was not significantly correlated with the dependent variables. Follow-up ANCOVAs showed the FraX group to have significantly reduced PV area relative to control subjects.

BRAIN ASYMMETRY - A significantly smaller Left $>$ Right amygdala asymmetry $(p=0.03)$ was observed in subjects with FraX $\left(\right.$ Left $=2.32 \mathrm{~cm}^{3}$; Right $\left.=2.24 \mathrm{~cm}^{3}\right)$ relative to control subjects $\left(\right.$ Left $=2.60 \mathrm{~cm}^{3} ;$ Right $\left.=2.37 \mathrm{~cm}^{3}\right)$. The FraX group $\left(\right.$ Left $=118.2 \mathrm{~cm}^{3} ;$ Right $=$ $\left.119.0 \mathrm{~cm}^{3}\right)$ also showed reduced Right $>$ Left frontal lobe asymmetry $(p=0.03)$ relative to control subjects $\left(\right.$ Left $=120.1 \mathrm{~cm}^{3}$; Right $\left.=122.8 \mathrm{~cm}^{3}\right)$. In the anterior cerebral cortex, asymmetry differences were observed for the anterior dorsal $\left(\right.$ FraX: Left $/$ Right $=7.5 / 7.7 \mathrm{~cm}^{3}$; control: $\left.6.3 / 6.8 \mathrm{~cm}^{3} ; p<0.03\right)$, anterior midsuperior (FraX: Left $/$ Right $=14.8 / 14.9 \mathrm{~cm}^{3}$; control: $\left.14.0 / 14.4 \mathrm{~cm}^{3} ; p<0.02\right)$, and anterior midinferior $\left(\right.$ FraX: Left $/$ Right $=16.0 / 16.2 \mathrm{~cm}^{3}$; control: $15.8 / 16.3 \mathrm{~cm}^{3} ; p=0.01$ ) cerebral regions. No other asymmetry differences were observed for other brain regions where significant bilateral group differences had been detected.

\section{Voxel-Based Morphometry}

Increased $\mathrm{GM}$ volume was detected in the bilateral $\mathrm{CN}$ and right fusiform gyrus of participants with FraX, predominantly in the $\mathrm{CN}$ head region (Table 4 and Fig 2). In the reverse contrast, VBM analysis demonstrated decreased GM volume in left orbital frontal cortex and left posterior STG in subjects with FraX (see Table 4). A second analysis using a 4mm smoothing kernel confirmed bilateral enlargement of the $\mathrm{CN}$ in the FraX group.

White matter VBM showed greater white matter volume in the right inferior frontal region in subjects with FraX, whereas control subjects showed greater white matter volume in bilateral superior temporal region.

\section{Surface-Based Anatomic Modeling}

The $\mathrm{CN}$ showed significant localized increases in radial size in the FraX group compared with control subjects (permutation tests: Left: $p<0.001$ [lateral CN surface], $p<0.002$ [medial CN surface]; Right: $p<0.02$ [lateral CN surface], $p=$ not significant [medial CN surface]). Figure 3 shows the surface maps of the percentage difference of $\mathrm{CN}$ size between FraX and control subjects (top row) and the statistical significance of the differences (bottom row). Significant increases in size in FraX compared with control subjects were primarily localized to the head of the caudate. The reverse contrast (control > FraX) did not yield any significant differences.

\section{Brain Regions That Distinguish Fragile X Syndrome from Control Subjects}

Eleven of the 16 brain region measures were significantly different between FraX and control subjects (see Table 3). To identify the brain regions that best distinguish between groups, we entered these 11 brain measures, adjusted for brain tissue volume, and age into the QROC as independent variables (Fig 4).

At the first level, the adjusted $\mathrm{CN}$ volume was identified as the optimal variable that distinguished between FraX and typically developing control subjects, with a cut point of $9.8 \mathrm{~cm}^{3}$. Thus, the adjusted $\mathrm{CN}$ volume was used to divide the full sample into two subsamples. The process was then repeated with three additional brain volumes identified distinguishing among subject subgroups: adjusted volumes of the PV, amygdala, and STG. Table 5 summarizes the combinations of the QROC partition groups and the sensitivity, specificity, and weighted $\kappa$ values of each combination. 


\section{Associations of Neuroanatomy with FMRP, Cognitive Deficit, and Aberrant Behavior}

Based on the results of the QROC, we assessed whether sizes (adjusted for brain tissue volume) of the caudate, PV, amygdala, and STG would be associated with FMRP levels, IQ, and aberrant behaviors. The resulting exploratory correlation analyses within the FraX and control groups were limited by missing data or analyses that were not applicable to control subjects (eg, FMRP levels were available for only 57 of 84 participants with FraX). In addition, control subjects did not have FMRP levels (which are assumed to be 100\%) or scores on the two behavior checklists that are designed for individuals with developmental disability.

For the FraX group, adjusted CN volumes negatively correlated with FMRP levels ( $\mathrm{n}=57 ; \rho$ $=-0.43 ; p<0.0001)$, FSIQ $(\mathrm{n}=83 ; \rho=-0.32 ; p=0.003)$, and positively correlated with total $\mathrm{ABC}(\mathrm{n}=63 ; \rho=0.33 ; p=0.009)$. There was a trend for adjusted $\mathrm{CN}$ volume to be correlated with total AbBC score as well $(\mathrm{n}=59 ; \rho=0.23 ; p<0.09)$. An analysis of the ABC and AbBC subscale scores showed that adjusted $\mathrm{CN}$ volume was significantly correlated with several dimensions of aberrant behavior across both measures including the Sensory $(\rho=0.26 ; p<$ $0.05)$, Body Object Use $(\rho=0.31 ; p<0.02)$, and Language $(\rho=0.33 ; p<0.01)$ subscales of the ABC, and the Stereotypy subscale of the AbBC $(\rho=0.32 ; p<0.02)$. Adjusted CN volume was not correlated with FSIQ in control subjects $(\mathrm{n}=70 ; \rho=0.08 ; p=0.53)$. Adjusted PV size positively correlated with FMRP levels $(\mathrm{n}=57 ; \rho=0.33 ; p<0.02)$ and FSIQ scores $(\mathrm{n}=83$; $\rho=0.35 ; p=0.001$ ) in the FraX group, but not with total ABC or Stereotypy Scale scores.

Subscale analysis showed that the adjusted PV size was significantly (negatively) correlated with the Body Object Use subscale of the ABC ( $\rho=-0.34 ; p<0.006)$. Adjusted PV size was not significantly correlated with FSIQ in control subjects $(\mathrm{n}=70 ; \rho=-0.04 ; p=0.74)$.

Unexpectedly, adjusted STG volume was positively correlated with total AbBC ( $\mathrm{n}=58 ; \rho=$ $0.29 ; p=0.03)$, AbBC Stereotypy subscale ( $\rho=0.37 ; p=0.005$ ), and ABC Body Object Use subscale $(\mathrm{n}=63 ; \rho=0.33 ; p<0.01)$ scores in the FraX group. No other significant correlations were observed between amygdala or STG volumes and FMRP, FSIQ, and the behavior scales for either group. Finally, linear multiple regression using all four (adjusted) regional variables as predictors and FSIQ as the outcome variable indicated that the $\mathrm{CN}(\beta=-0.34 ; p=0.001)$, PV $(\beta=0.39 ; p<0.0001)$, and STG $(\beta=0.25 ; p<0.02)$ all had significant, independent effects on FSIQ in the FraX group, accounting for $31 \%$ of the total variance in this measure $(\mathrm{F}=8.7$; $p<0.0001)$.

Surface-based correlation maps were used to further investigate the association of aberrant $\mathrm{CN}$ morphology in FraX with both aberrant behavior and FMRP levels (Fig 5). The maps demonstrate that in regions of the right lateral and right medial caudate, radial expansions are negatively correlated with FMRP levels ( $p<0.01$, corrected) and positively correlated with total ABC $(p<0.05$, corrected) and AbBC Stereotypy scores $(p<0.05$, corrected). Correlations for the left $\mathrm{CN}$ did not reach statistical significance.

\section{Discussion}

In this study, we combined multiple complementary image processing methods, volumetric analyses, VBM, and surface-based anatomic modeling, to demonstrate a discernible neuroanatomic phenotype associated with FraX. Several brain regions were identified as morphologically aberrant in this large group of children with FraX, most prominently, the CN, $\mathrm{PV}$, amygdala, and STG. Of these regions, the CN and PV were correlated with the severity of FMRP deficiency or cognitive deficit in children with FraX. In addition, significant associations were demonstrated between $\mathrm{CN}$ volume and severity of aberrant behaviors.

Our hypothesis regarding abnormal $\mathrm{CN}$ development and its association to FMRP and severity of cognitive and behavioral symptoms was strongly supported. Gross caudate volumes in male 
subjects with FraX were more than $20 \%$ larger than age-matched male control subjects, and increased size of this region was confirmed in the VBM analyses. The CN enlargement in FraX was especially strong early in development. These results replicate and significantly extend previous findings that showed an association among $\mathrm{CN}$ volume, $F M R l$ gene inactivation (which is highly correlated with FMRP levels), and lower FSIQ. ${ }^{8}$ Interestingly, increased CN volumes have also been detected in non-FraX children and adults with autism. ${ }^{26-28}$ In one study of autism, increased $\mathrm{CN}$ volumes were associated with repetitive behaviors. ${ }^{27}$

Furthermore, and in line with results from the surface mapping correlations presented here, enlargement and altered metabolism of the $\mathrm{CN}$ in autism have been primarily localized to the right side. ${ }^{27,29}$ When combined with findings from this study, these results suggest that abnormal neurodevelopment of the $\mathrm{CN}$ may be a common pathway leading to a phenotype that includes autistic and stereotypic behaviors.

Our hypothesis regarding the PV in FraX was largely supported. In line with previous studies, 7,30 the region of interest analysis indicated that individuals with FraX had significantly reduced PV sizes (approximately 20\% in male relative to control subjects). Furthermore, FMRP level positively correlated and FSIQ scores negatively correlated with PV size, suggesting that more abnormal PV development is linked to more severe cognitive deficits in FraX. Though associations between PV size and aberrant behaviors were not as prominent as $\mathrm{CN}$-behavior correlations, decreasing PV size was correlated with increasing scores on the ABC subscale that includes the most items related to stereotypic behaviors (Body and Object Use). This finding is consistent with a previous study of school-age girls with $\mathrm{FraX}^{31}$ where PV size was negatively correlated with stereotypic behaviors, and to a more recent study of non-FraX children with autism ${ }^{32}$ where the size of lobules VI-VII within the PV was found to be negatively correlated with exploratory and stereotypic behavior.

Our hypotheses regarding the amygdala and STG were partially supported. As hypothesized, both regions were smaller in FraX compared with control subjects, and both were identified by the QROC to distinguish between FraX and control subjects. Portions of the left (GM and white matter) and right STG (white matter only) were also identified as smaller in FraX with VBM. However, no associations were detected between the size of these regions and FMRP levels or FSIQ.

The unanticipated correlation of STG volume with behavioral symptoms is difficult to explain because it occurred in the direction opposite to that expected. However, three of the primary variables of interest, including the STG, independently contributed to cognitive outcome in subjects with FraX in the expected direction (eg, larger STG indicates higher IQ); taken together, the volumes of these regions accounted for more than $30 \%$ of the variance in FSIQ. These findings suggest that abnormal neurodevelopment of the CN, PV, and STG contributes to the pathogenesis of cognitive deficits in FraX. These results are also in line with accumulating evidence for the role of these brain structures in higher order cognitive functions including language, visuospatial domains, attention, and executive functions. ${ }^{33,34}$

Previous studies have suggested that brain morphology in FraX is altered, with particular emphasis on abnormal prefrontal-striatal neuroanatomy. $8,13,14$ However, the findings of this study provide a more specific picture of abnormal neuroanatomic circuits in FraX. For example, both VBM and surface-based anatomic modeling localized the enlargement of the CN in FraX to lateral and medial parts of the $\mathrm{CN}$ head region. The head of the $\mathrm{CN}$ is a component of two major corticostriatothalamic circuits. ${ }^{33,35}$ One circuit connects the dorsolateral prefrontal cortex with the dorsolateral head of $\mathrm{CN}$ and is involved in executive functioning. The second circuit connects the lateral orbitofrontal cortex with the ventromedial head of caudate. ${ }^{33,35}$ Dysfunction of this second circuit is associated with perseverative behavior. ${ }^{35}$ The clinical phenotype of FraX is highly consistent with impairments of these two corticostriatal circuits 
as executive functioning deficits and perseverative behaviors are common features of this neurogenetic condition. 5,36

This study also provides new evidence for abnormal orbitofrontal-amygdala circuitry in FraX. Although FMRl knock-out mice demonstrate evidence of amygdala dysfunction including audiogenic seizures and an abnormal conditioned fear response, ${ }^{37,38}$ to our knowledge, this is the first study to report amygdala and orbitofrontal anatomic abnormalities in a group with the FMR1 full mutation. Orbitofrontal-amygdala circuitry participates in regulation of social behavior and affect. ${ }^{39}$ Though dysfunction of this circuit may be relevant to clinical features of fragile $\mathrm{X}$ such as social anxiety and withdrawal and reduced eye contact, ${ }^{4}$ we note that, in this study, we did not find an association between amygdala volume and behavioral measures.

The VBM and region of interest approach converged to demonstrate reduced STG volume in FraX, previously shown in a much smaller sample. ${ }^{40}$ These STG structural abnormalities in FraX are in line with functional magnetic resonance imaging findings showing that subjects with FraX have decreased activation of the superior temporal region during processing of face and gaze stimuli. ${ }^{41}$ The increase in fusiform gyrus volume detected by the VBM approach might also be related to decreased specificity in activation of this region found in FraX subjects in response to direct gaze. ${ }^{41}$ Specifically, fusiform enlargement in FraX could be related to lack of maturation and refinement of this face-processing region in affected individuals secondary to gaze aversion and aberrant functional specialization. We are currently collecting specific eye movement data in all new subjects undergoing imaging to further explore this issue.

The lack of complete convergence between VBM and volumetric results in this study could be influenced by tissue segmentation issues. For example, VBM size differences will be more readily identified in structures that have greater tissue homogeneity, such as the $\mathrm{CN}$, than in other regions, such as the amygdala, that contain a greater admixture of GM and white matter.

In this study, we utilized a statistical method (QROC) that addresses limitations of traditional parametric statistical analyses commonly used in analyzing brain imaging data. ${ }^{25,42}$ In providing a hierarchical profile of neuroanatomy associated with FraX, the QROC identified a combination of large $\mathrm{CN}$, small PV, small amygdala, and small STG that at specific cut-point values distinguished between the FraX and control groups with $92 \%$ sensitivity and $61 \%$ specificity. These results suggest that QROC may be a valuable tool to identify combinations of brain regions that can distinguish between patients and control subjects.

A potential limitation of our study is aggregating data from different scanners over time, as this raises the question of compatibility of imaging data. Though there were differences in the male/female ratio and mean ages of subjects from the two sites, these differences did not appear to influence the observed between-group findings. The likelihood of a type 1 error resulting from more than one imaging site is also reduced given comparable image characteristics and morphometric results when analyses were broken out by location (see supplementary materials). We also controlled for key image acquisition parameters that are important for maintaining image compatibility across sites. ${ }^{21}$ Both sites had similar scanner type, slice orientation (coronal), thin slice thickness, field of view, and acquisition matrix size. As shown in a previous study, these parameters are key in preserving image compatibility between sites. ${ }^{21}$ For the volumetric measures, the approach used for delineating and subdividing the anterior cerebral cortex was limited by the fact that this method did not rely on sulcal/gyral landmarks or on functionally relevant landmarks.

Similarly, other approaches for performing the surface-based modeling procedure could have been used. As a requirement of the method used here, caudate region of interests were first realigned into a standard anatomic space that retained the roughly tubular shape of this structure 
in the anterior-posterior direction. As an alternative to this method, one could compute the set of points that are the local maxima of three-dimensional distance to the boundary and connect them. A smooth curve could then be computed iteratively with maximal three-dimensional distance to the boundary surface, by numeric optimization or solving boundary-based partial differential equations. ${ }^{43,44}$ The resulting three-dimensional distance map is intrinsic and does not depend on the orientation of the structure or how it is aligned or sliced. It is not necessarily more accurate to compute the medial curve in this way, but it is rotation invariant and avoids the need to first align the structure into a standard orientation, which may add some small but measurable error associated with rotational errors in registration.

Another limitation is the large cognitive discrepancy between the FraX group and healthy control subjects. Future studies should include cognitively matched control subjects with other forms of developmental disabilities and children with autism (without FraX) to more definitively identify the neuroanatomic phenotype associated with FraX.

Additional brain regions of potential interest to understanding the pathophysiology of brain dysfunction in FraX were also identified. Individuals with FraX in this study demonstrated an aberrant profile of anterior cerebral brain morphology with reduced size of ventral regions and relatively increased size of the dorsal region. The ventrodorsal (smaller to bigger) "gradient" of size in the FraX anterior cerebral cortex is of opposite polarity to that observed in Williams' syndrome, a neurogenetic condition associated with a contiguous deletion on chromosome 7q. 45 This finding is remarkable because individuals with Williams' syndrome also demonstrate a social behavioral phenotype that is of opposite polarity to that observed in FraX: appetitive as opposed to avoidant. When combined with results showing that frontal and temporal lobes are smaller and parietal and occipital lobes are larger in FraX relative to control subjects, the atypical anterior_cerebral morphology observed here suggests that FMRP may bind to and regulate the translation of messenger RNA important for cortical gradients and shape.

In summary, using comprehensive and complementary analytic approaches, we were able to identify intriguing links between the presence of the FMRI full mutation and abnormal development of the brain in FraX, in particular the CN and PV. We also demonstrate the potential role of specific brain regions in the pathogenesis of cognitive deficits and aberrant behavior symptoms in FraX. Being a causatively well-defined syndrome, FraX is a compelling model from which to learn about the intricate and complex linkages among measurable factors relating to gene, brain, and cognition.

\section{Supplementary Material}

Refer to Web version on PubMed Central for supplementary material.

\section{Acknowledgments}

This study was supported by the NIMH (MH50047, MH01142), NICHD (HD31715) and the "Lynda and Scott Canel Fund for Fragile X Research" (A.L.R.), and by the NIBIB (EB01651) and NCRR (RR019771) (P.M.T.), and NARSAD Young Investigator Award (D.G.).

\section{References}

1. Happe F, Ronald A, Plomin R. Time to give up on a single explanation for autism. Nat Neurosci 2006;9:1218-1220. [PubMed: 17001340]

2. Belmonte MK, Bourgeron T. Fragile $X$ syndrome and autism at the intersection of genetic and neural networks. Nat Neurosci 2006;9:1221-1225. [PubMed: 17001341]

3. Zalfa F, Achsel T, Bagni C. mRNPs, polysomes or granules: FMRP in neuronal protein synthesis. Curr Opin Neurobiol 2006;6:265-269. [PubMed: 16707258] 
4. Reiss AL, Dant CC. The behavioral neurogenetics of fragile X syndrome: analyzing gene-brainbehavior relationships in child developmental psychopathologies. Dev Psychopathol 2003;15:927968. [PubMed: 14984133]

5. Hagerman, RJ.; Hagerman, PJ. Fragile X syndrome: diagnosis, treatment and research. Vol. 3rd ed. John Hopkins University Press; Baltimore: 2002.

6. Hessl D, Rivera SM, Reiss AL. The neuroanatomy and neuroendocrinology of fragile X syndrome. Ment Retard Dev Disabil Res Rev 2004;10:17-24. [PubMed: 14994284]

7. Mostofsky SH, Mazzocco MM, Aakalu G, et al. Decreased cerebellar posterior vermis size in fragile X syndrome: correlation with neurocognitive performance. Neurology 1998;50:121-130. [PubMed: 9443468]

8. Reiss AL, Abrams MT, Greenlaw R, et al. Neurodevelopmental effects of the FMR-1 full mutation in humans. Nat Med 1995;1:159-167. [PubMed: 7585014]

9. Barnea-Goraly N, Eliez S, Hedeus M, et al. White matter tract alterations in fragile X syndrome: preliminary evidence from diffusion tensor imaging. Am J Med Genet B Neuropsychiatr Genet 2003;118:81-88. [PubMed: 12627472]

10. Hoeft F, Hernandez A, Parthasarathy S, et al. Fronto-striatal dysfunction and potential compensatory mechanisms in male adolescents with fragile X syndrome. Hum Brain Mapp 2007;28:543-554. [PubMed: 17437282]

11. Menon V, Leroux J, White CD, Reiss AL. Frontostriatal deficits in fragile X syndrome: relation to FMR1 gene expression. Proc Natl Acad Sci U S A 2004;101:3615-3620. [PubMed: 14993603]

12. Palmen SJ, van Engeland $H$. Review on structural neuroimaging findings in autism. J Neural Transm 2004;111:903-929. [PubMed: 15206006]

13. Eliez S, Blasey CM, Freund LS, et al. Brain anatomy, gender and IQ in children and adolescents with fragile X syndrome. Brain 2001;124:1610-1618. [PubMed: 11459752]

14. Kates WR, Folley BS, Lanham DC, et al. Cerebral growth in Fragile X syndrome: review and comparison with Down syndrome. Microsc Res Tech 2002;57:159-167. [PubMed: 12112452]

15. Achenbach, T. Manual for the Child Behavior Checklist/4-18 and 1991 profile. Department of Psychiatry, University of Vermont; Burlington, VT: 1991.

16. Kates WR, Abrams MT, Kaufmann WE, et al. Reliability and validity of MRI measurement of the amygdala and hippocampus in children with fragile X syndrome. Psychiatry Res 1997;75:31-48. [PubMed: 9287372]

17. Oberle I, Rousseau F, Heitz D, et al. Instability of a 550-base pair DNA segment and abnormal methylation in fragile X syndrome. Science 1991;252:1097-1102.

18. Willemsen R, Oosterwijk JC, Los FJ, et al. Prenatal diagnosis of fragile X syndrome. Lancet 1996;348:967-968. [PubMed: 8843844]

19. Wadden NP, Bryson SE, Rodger RS. A closer look at the Autism Behavior Checklist: discriminant validity and factor structure. J Autism Dev Disord 1991;21:529-541. [PubMed: 1778965]

20. Aman MG, Singh NN, Stewart AW, Field CJ. Psychometric characteristics of the Aberrant Behavior Checklist. Am J Ment Defic 1985;89:492-502. [PubMed: 3158201]

21. Patwardhan AJ, Eliez S, Warsofsky IS, et al. Effects of image orientation on the comparability of pediatric brain volumes using three-dimensional MR data. J Comput Assist Tomogr 2001;25:452457. [PubMed: 11351198]

22. Good CD, Johnsrude IS, Ashburner J, et al. A voxel-based morphometric study of ageing in 465 normal adult human brains. Neuroimage 2001;14:21-36. [PubMed: 11525331]

23. Ashburner J, Friston KJ. Voxel-based morphometry-the methods. Neuroimage 2000;11:805-821. [PubMed: 10860804]

24. Frisoni GB, Sabattoli F, Lee AD, et al. In vivo neuropathology of the hippocampal formation in AD: A radial mapping MR-based study: a radial mapping MR-based study. Neuroimage 2006;32:104110. [PubMed: 16631382]

25. Kraemer, HC. Evaluating medical tests: objective and quantitative guidelines. Sage Publications; Newbury Park, CA: 1992. 
26. Haznedar MM, Buchsbaum MS, Hazlett EA, et al. Volumetric analysis and three-dimensional glucose metabolic mapping of the striatum and thalamus in patients with autism spectrum disorders. Am $\mathrm{J}$ Psychiatry 2006;163:1252-1263. [PubMed: 16816232]

27. Hollander E, Anagnostou E, Chaplin W, et al. Striatal volume on magnetic resonance imaging and repetitive behaviors in autism. Biol Psychiatry 2005;58:226-232. [PubMed: 15939406]

28. Sears LL, Vest C, Mohamed S, et al. An MRI study of the basal ganglia in autism. Prog Neuropsychopharmacol Biol Psychiatry 1999;23:613-624. [PubMed: 10390720]

29. Levitt JG, O’Neill J, Blanton RE, et al. Proton magnetic resonance spectroscopic imaging of the brain in childhood autism. Biol Psychiatry 2003;54:1355-1366. [PubMed: 14675799]

30. Kaufmann WE, Cooper KL, Mostofsky SH, et al. Specificity of cerebellar vermian abnormalities in autism: a quantitative magnetic resonance imaging study. J Child Neurol 2003;18:463-470. [PubMed: 12940651]

31. Mazzocco MM, Kates WR, Baumgardner TL, et al. Autistic behaviors among girls with fragile X syndrome. J Autism Dev Disord 1997;27:415-435. [PubMed: 9261667]

32. Pierce K, Courchesne E. Evidence for a cerebellar role in reduced exploration and stereotyped behavior in autism. Biol Psychiatry 2001;49:655-664. [PubMed: 11313033]

33. Masterman DL, Cummings JL. Frontal-subcortical circuits: the anatomic basis of executive, social and motivated behaviors. J Psychopharmacol 1997;11:107-114. [PubMed: 9208374]

34. Rapoport M, van Reekum R, Mayberg H. The role of the cerebellum in cognition and behavior: a selective review. J Neuropsychiatry Clin Neurosci 2000;12:193-198. [PubMed: 11001597]

35. Alexander GE, DeLong MR, Strick PL. Parallel organization of functionally segregated circuits linking basal ganglia and cortex. Annu Rev Neurosci 1986;9:357-381. [PubMed: 3085570]

36. Cornish KM, Munir F, Cross G. Differential impact of the FMR-1 full mutation on memory and attention functioning: a neuropsychological perspective. J Cogn Neurosci 2001;13:144-150. [PubMed: 11224914]

37. Chen L, Toth M. Fragile X mice develop sensory hyperreactivity to auditory stimuli. Neuroscience 2001;103:1043-1050. [PubMed: 11301211]

38. Musumeci SA, Bosco P, Calabrese G, et al. Audiogenic seizures susceptibility in transgenic mice with fragile X syndrome. Epilepsia 2000;41:19-23. [PubMed: 10643918]

39. Adolphs R. The neurobiology of social cognition. Curr Opin Neurobiol 2001;11:231-239. [PubMed: 11301245]

40. Reiss AL, Lee J, Freund L. Neuroanatomy of fragile X syndrome: the temporal lobe. Neurology 1994;44:1317-1324. [PubMed: 8035938]

41. Garrett AS, Menon V, MacKenzie K, Reiss AL. Here's looking at you, kid: neural systems underlying face and gaze processing in fragile X syndrome. Arch Gen Psychiatry 2004;61:281-288. [PubMed: 14993116]

42. Kiernan M, Kraemer HC, Winkleby MA, et al. Do logistic regression and signal detection identify different subgroups at risk? Implications for the design of tailored interventions. Psychol Methods 2001;6:35-48. [PubMed: 11285811]

43. Bansal R, Staib LH, Xu D, et al. Statistical analyses of brain surfaces using Gaussian random fields on 2-D manifolds. IEEE Trans Med Imaging 2007;26:46-57. [PubMed: 17243583]

44. Yushkevich PA, Zhang H, Gee JC. Statistical modeling of shape and appearance using the continuous medial representation. Med Image Comput Comput Assist Interv Int Conf Med Image Comput Comput Assist Interv 2005;8:725-732. [PubMed: 16686024]

45. Reiss AL, Eckert MA, Rose FE, et al. An experiment of nature: brain anatomy parallels cognition and behavior in Williams syndrome. J Neurosci 2004;24:5009-5015. [PubMed: 15163693] 


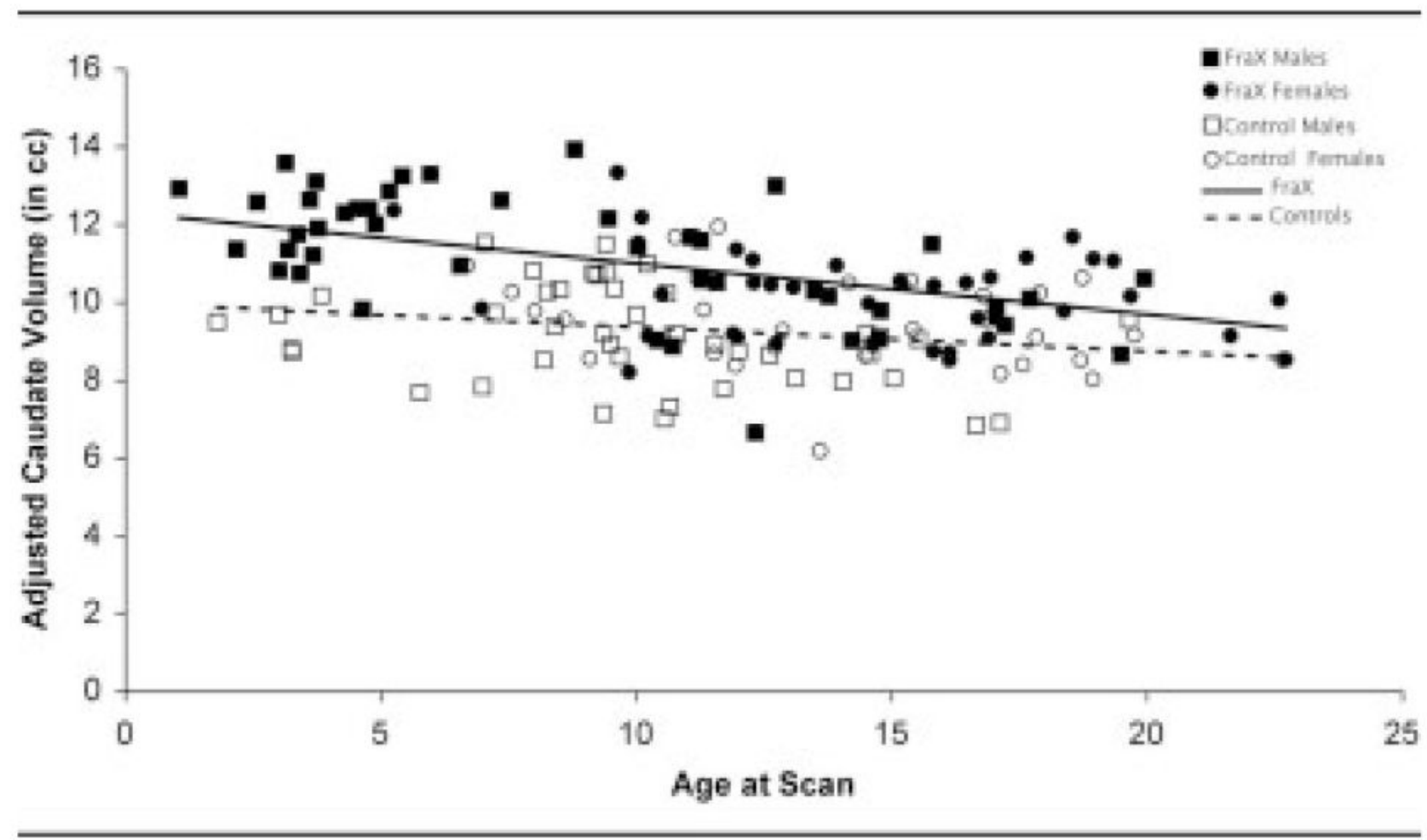

Fig 1.

Caudate nuclei volumes, adjusted for brain tissue volume, in fragile X syndrome and in typically developing control subjects across development. Solid squares denote male individuals with fragile $X$ syndrome; solid circles denote female individuals with fragile $\mathrm{X}$ syndrome; open squares denote male control subjects; open circles denote female control subjects; solid line indicates fragile $X$ syndrome; dotted line indicates control (no fragile $X$ syndrome). 


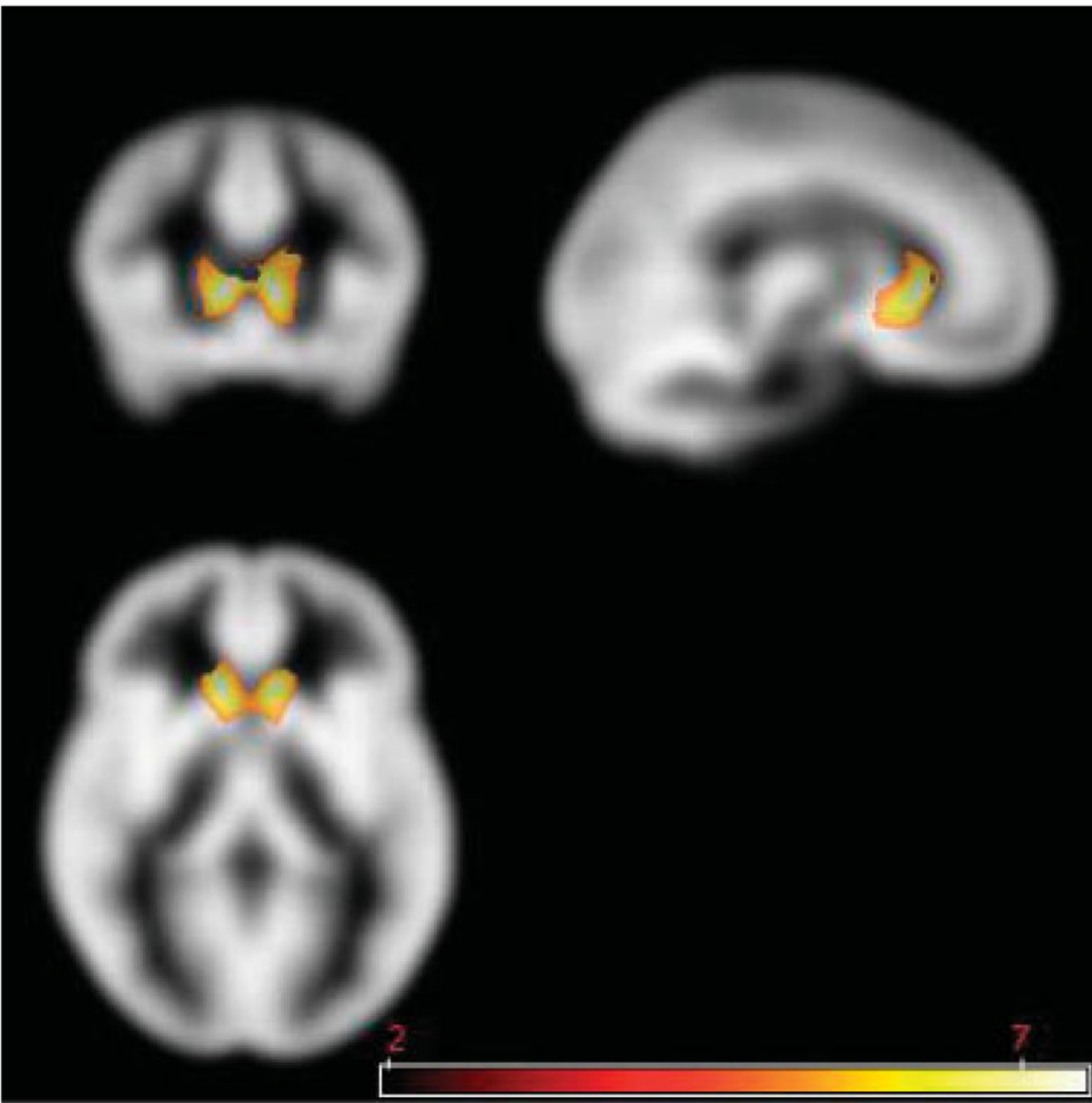

Fig 2.

Regions of increased gray matter volume in subjects with fragile X syndrome compared with typically developing control subjects using voxel-based morphometry. The area highlighted in yellow-white corresponds to caudate nucleus regions showing significantly greater gray matter volume in fragile X compared with control subjects. Statistical Parametric Mapping (SPM) overlaid on Cincinnati Children's Hospital Medical Center (CCHMC) template. Scale bar indicates $\mathrm{T}$ values. $\mathrm{p}=0.05$, family-wise error corrected; extent threshold $=10$. 


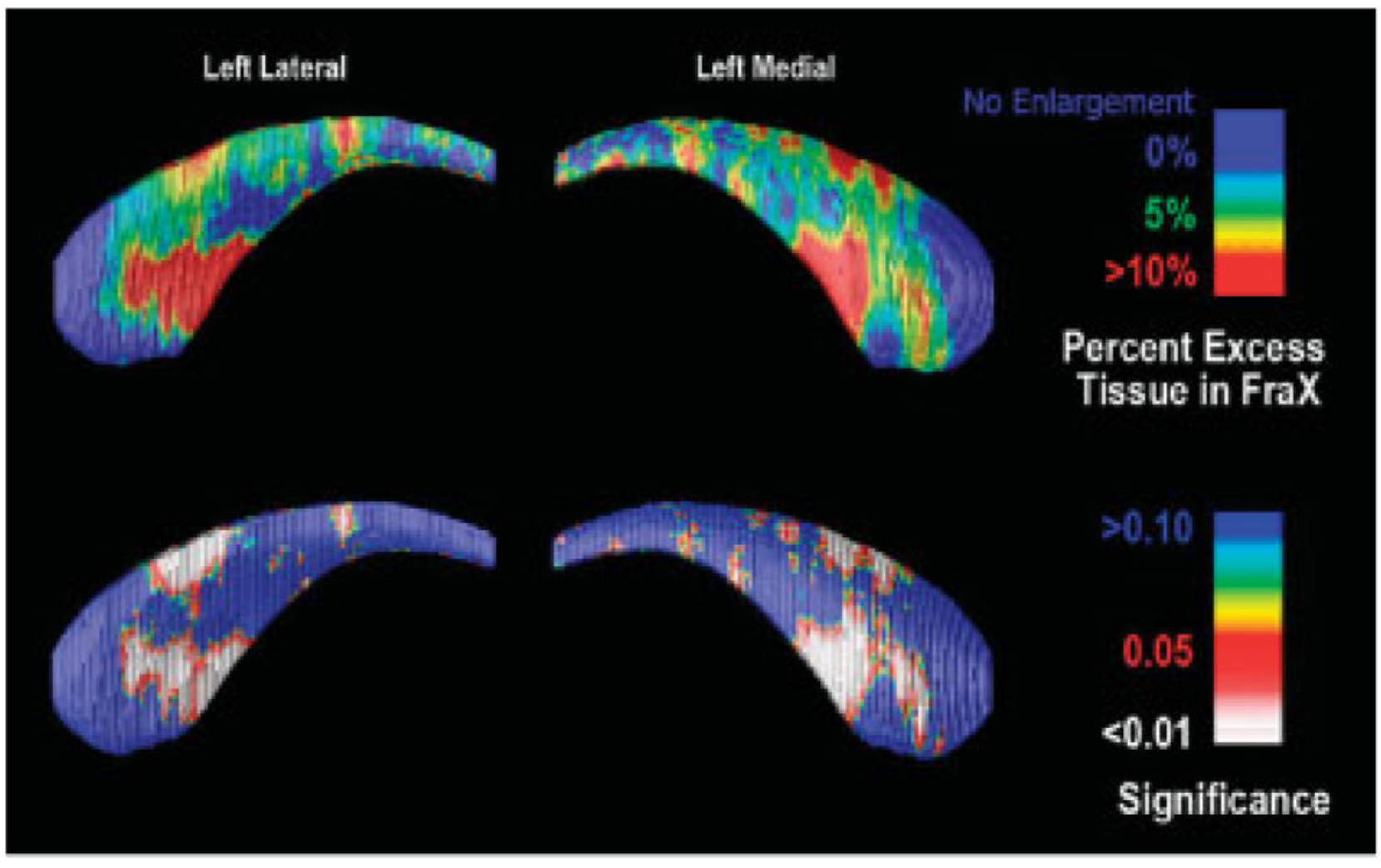

Fig 3.

Surface-based maps of the caudate nucleus $(\mathrm{CN})$. (top row) Local percentage difference in $\mathrm{CN}$ size between fragile $\mathrm{X}$ syndrome (FraX) and control subjects (top row). The ventral $\mathrm{CN}$ head shows systematic enlargement in FraX subjects, with an average 10\% radial expansion compared with control subjects. The significance of the group differences in radial extent is plotted (bottom row) detecting extensive enlargement of the $\mathrm{CN}$ head. 


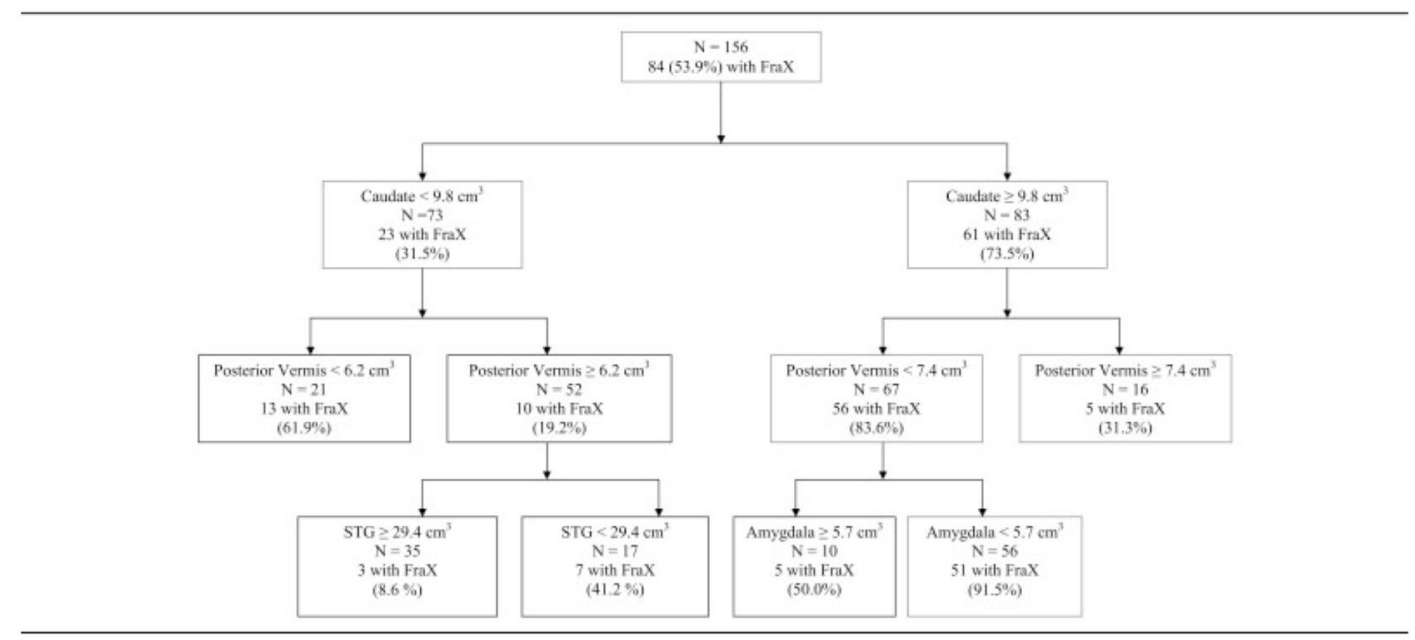

Fig 4.

Receiver operating characteristic curve showing the brain regions that best distinguish between subjects with fragile $\mathrm{X}$ syndrome (FraX) and typically developing control subjects. The caudate was identified first (73.5\% of subjects with large caudate had FraX), posterior vermis was identified second, and amygdala third. Of 56 subjects with small amygdala and small posterior vermis and large caudate, $91.5 \%$ had FraX, indicating that the combination of the three neuroanatomic abnormalities distinguishes between FraX and control subjects with specificity of $91.5 \%$ (see Table 5). STG = superior temporal gyrus. 


\section{a. FMRP}
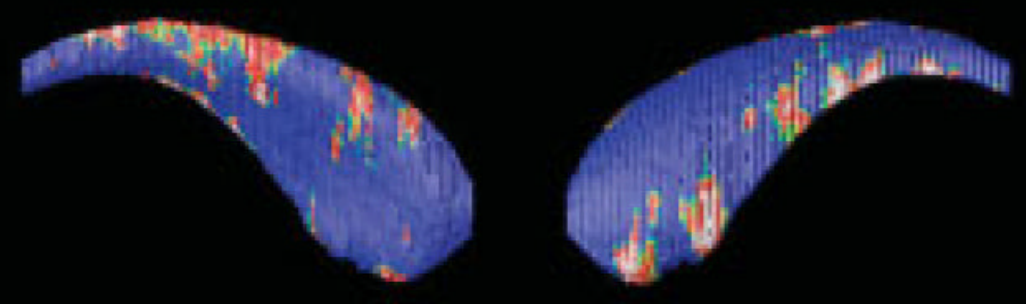

\section{b. Autism Behavior Checklist Scores}

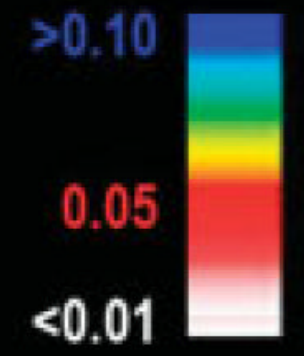

\section{c. Stereotypy Scores}

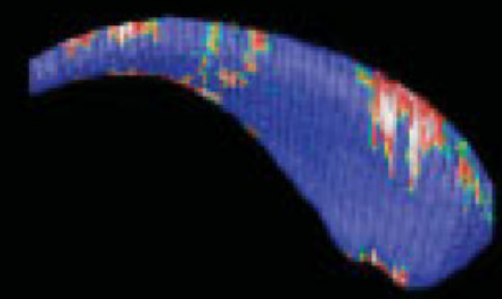

Right Lateral

\section{Significance}

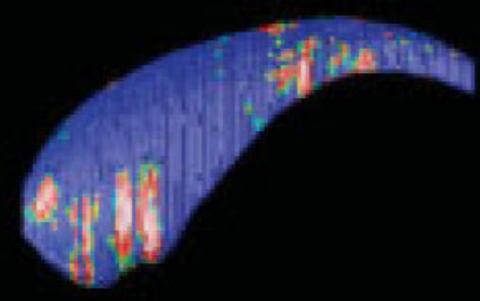

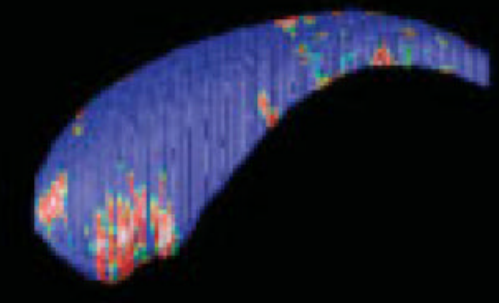

Right Medial

Fig 5.

Surface-based correlation maps showing a significant association between regions of radial caudate nucleus expansion and (A) reduced fragile X mental retardation protein (FMRP) levels, (B) Autism Behavior Checklist scores, and (C) Aberrant Behavior Checklist Stereotypy Subscale scores. 


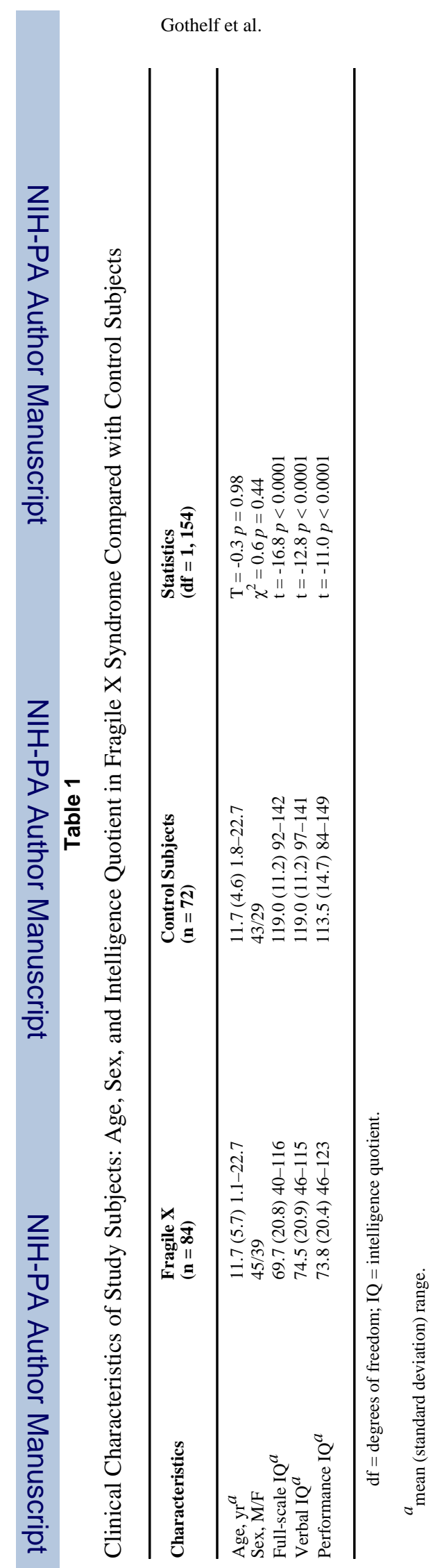

Ann Neurol. Author manuscript; available in PMC 2009 November 4. 


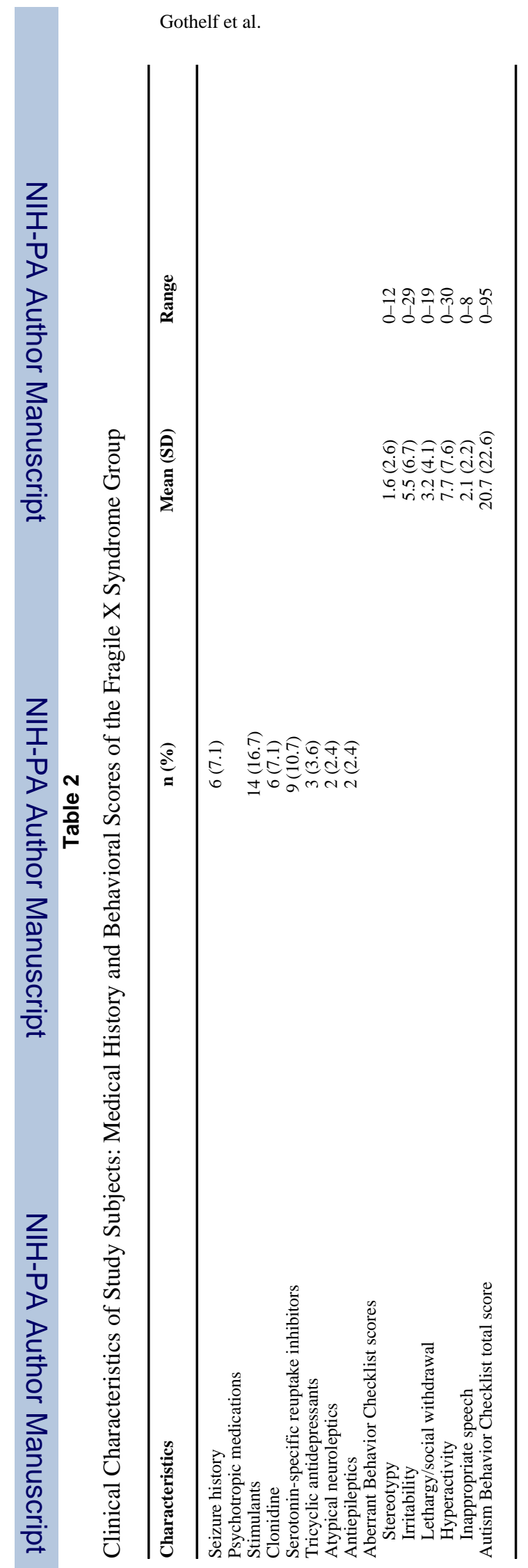

Ann Neurol. Author manuscript; available in PMC 2009 November 4. 


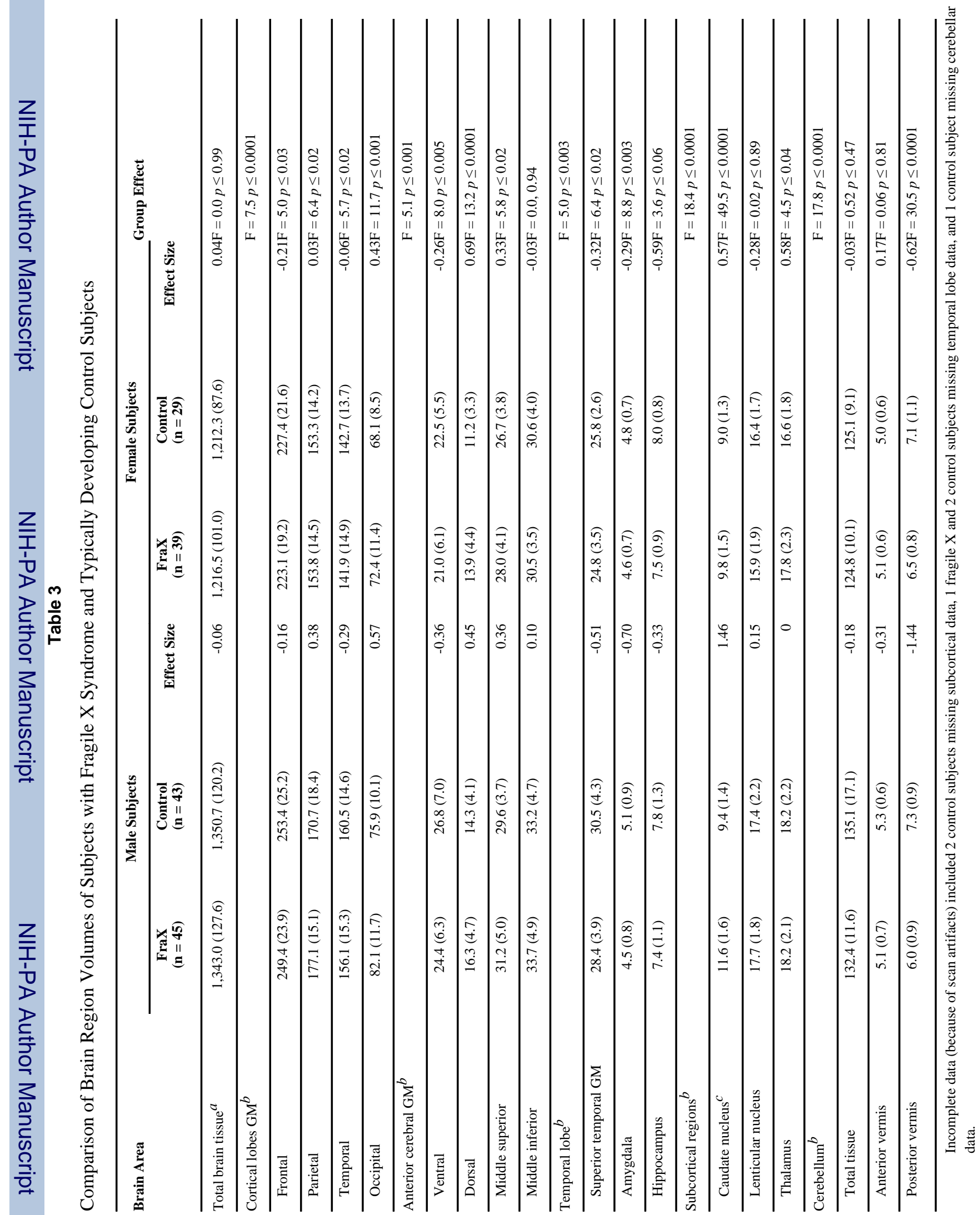




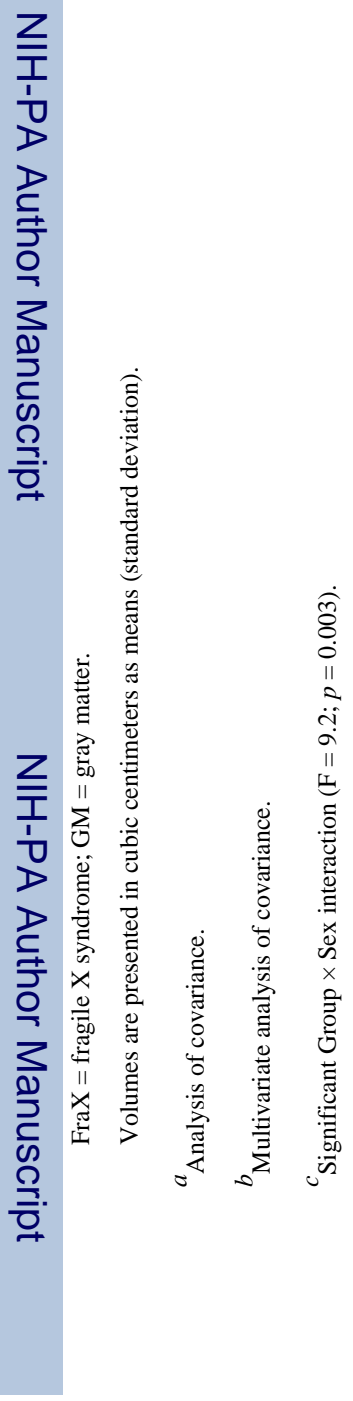


Gothelf et al.

Page 22

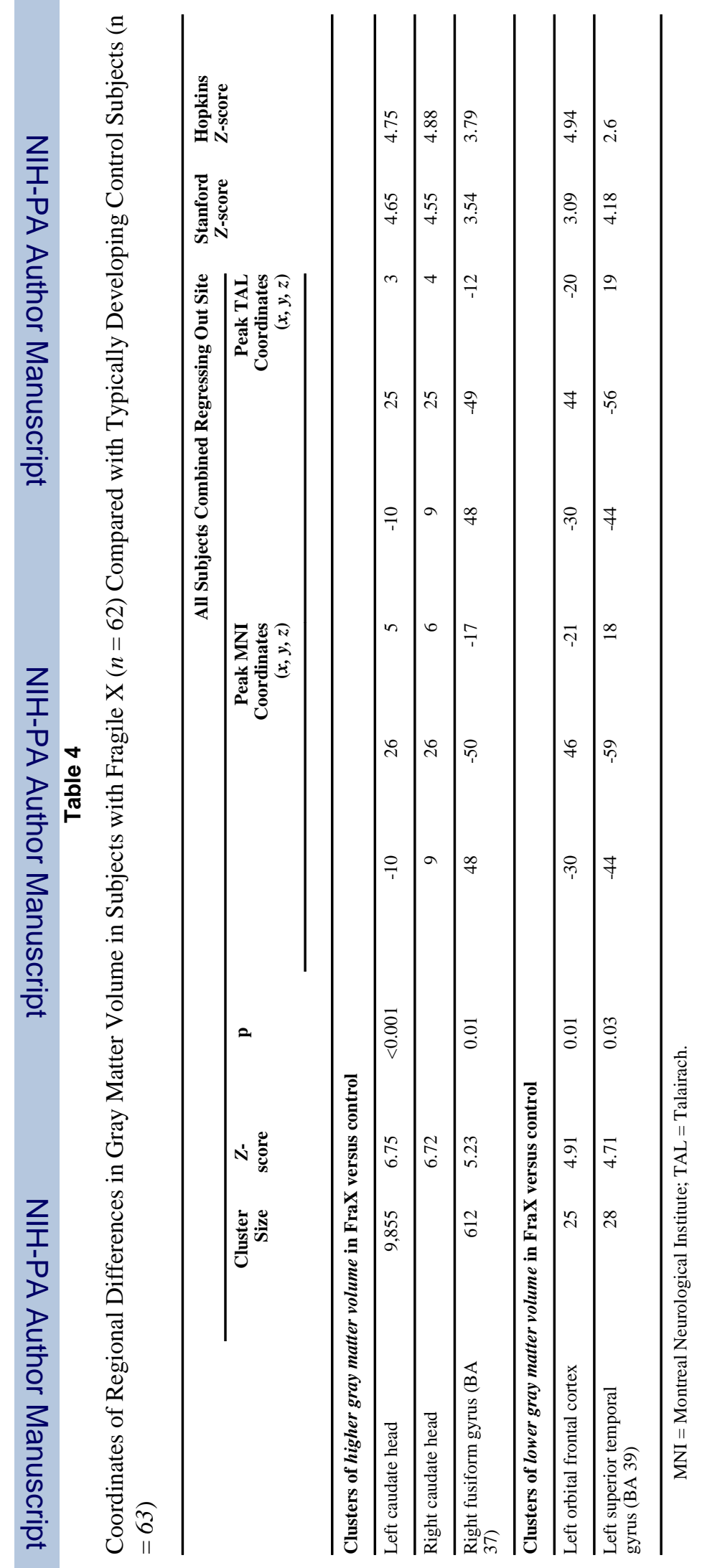

Ann Neurol. Author manuscript; available in PMC 2009 November 4. 


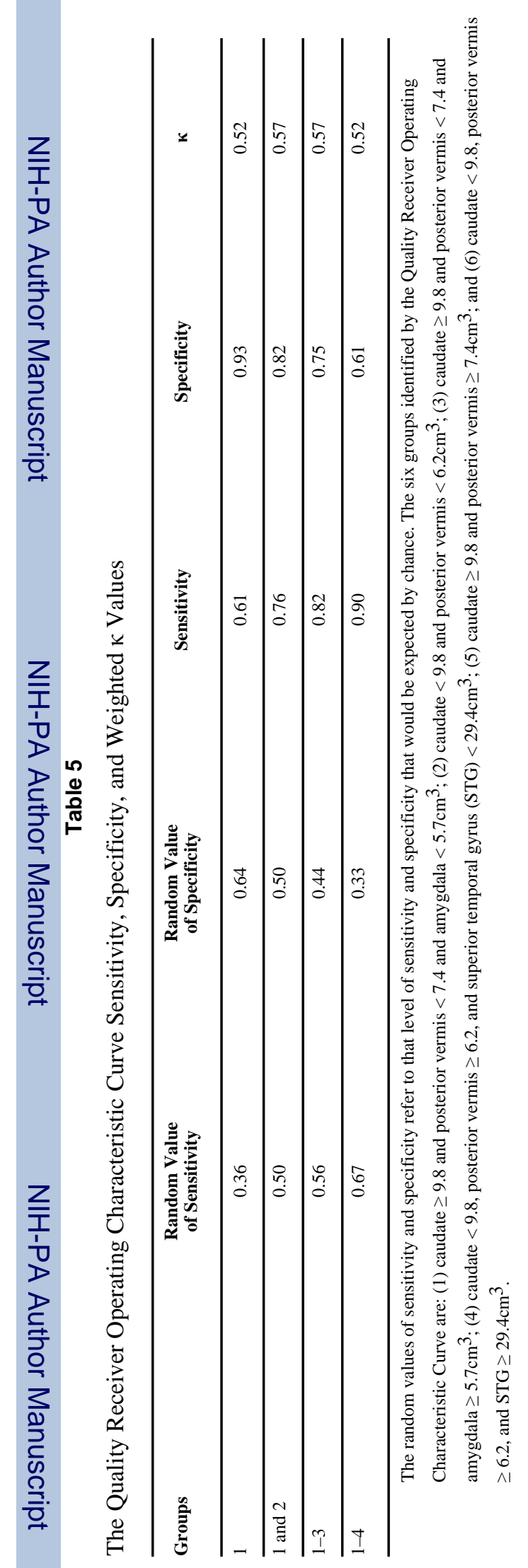

Ann Neurol. Author manuscript; available in PMC 2009 November 4. 